\title{
Utility of stereoelectroencephalography in preoperative assessment of temporal lobe epilepsy
}

Departments of
Clinical
Neurophysiology and
Neurosurgery,
Maudsley Hospital,
London, UK
C D Binnie
R D C Elwes
C E Polkey
A Volans
Correspondence to:
Dr Binnie, Neurosciences
Deparment, Maudsley
Hospital, Denmark Hill,
London SE5 8AZ, UK
Received 26 June 1992 and
in final revised form
9 November 1992.
Accepted 19 November 1992

\begin{abstract}
Of 269 consecutive patients entered into a preoperative assessment programme for possible surgical treatment of epilepsy, 33 had intracranial recording (SEEG) with combined subdural and depth electrodes for the purpose of localising a suspected temporal site of seizure onset. The findings in these patients are analysed with particular reference to: 1 ) the criteria of selection for SEEG and their validity; 2) information on SEEG compared with that obtained by less invasive means, including foramen ovale telemetry; 3) information on the use of intracerebral electrodes compared with subdural placements; 4) possible predictors of failure of localisation by SEEG and of surgical outcome. It was concluded that SEEG had usefully contributed to the management of $69 \%$ of the patients in whom it was used, establishing a previously unidentified site of seizure onset in $33 \%$, correcting an erroneous localisation in $15 \%$, and establishing inoperability in $21 \%$ of patients. No predictors of failure of SEEG or of surgery emerged; thus there was no evidence of unnecessary use of this procedure. Five patients were found with incorrect lateralisation of seizure onset on foramen ovale recording (of a total of 192 foramen ovale telemetries). Localisation of the ictal onset zone either by the distribution of inter-ictal discharges or by the initial ictal changes at subdural electrodes was unreliable, confirming the need for ictal, depth recordings.
\end{abstract}

( $¥$ Neurol Neurosurg Psychiatry 1994;57:58-65)

Successful treatment of epilepsy by resective surgery depends on removal of tissue which is both structurally and functionally abnormal and within which ictal activity is initiated. Despite recent developments in functional neuroimaging, electrophysiology remains the most important means of identifying such localised dysfunction.

In 1986, an international survey of centres engaged in surgical treatment of epilepsy ${ }^{1}$ indicated marked variations in the use of electrophysiology in preoperative assessment. Techniques have long been available for identifying sites of seizure onset by stereoelectroencephalography (SEEG), using stereotactically positioned intracerebral electrodes, sometimes in combination with subdural contacts. However, in the past many centres have achieved good results without intracranial recording, except acute electrocorticography ${ }^{2-4}$; some continue to do $\mathrm{so}^{5}$ or use SEEG rarely. Differences of approach may reflect differing patient populations rather than conflicting philosophies. Resective procedures in programmes making little or no use of invasive assessment are, or were, performed mainly on the temporal lobes, for mesial temporal sclerosis in particular. ${ }^{4-8}$ The feasibility of identifying the epileptogenic zone from the scalp EEG in these patients is clearly demonstrated, both by the very acceptable surgical results reported from centres not using SEEG, and by confirmation of EEG localisation by intracranial ictal recording in similar subjects. ${ }^{9}$ Other workers have emphasised rather the lack of agreement between scalp and depth recording. ${ }^{1011}$ Recently, however, there has been some convergence of practice, and most centres employ SEEG in 10-40\% of patients. ${ }^{12}$

Uncertainty remains about criteria for SEEG and concerning the choice of technology, specifically whether complex partial seizures should be investigated with subdural or depth electrodes, or with a combination of these.

Identification of the epileptogenic zone is only one requirement for successful treatment; surgical outcome is the criterion by which preoperative assessment should be judged; in practice this is difficult. Because of the differing patient populations and surgical techniques it is hard to compare results from centres with different programmes; indeed a feed-back seems to operate whereby the selection criteria, and hence the case-mix, in different centres are adjusted in such a way as to obtain very similar results overall. However, a single centre controlled trial of alternative programmes presents insuperable ethical problems, as this would necessarily require the surgeon concerned to act against his own judgment, by performing apparently unnecessary invasive recording, or operating with electrophysiological data considered inadequate.

The best evidence concerning indications for invasive neurophysiology is probably that gained by clinical audit in centres following defined preoperative protocols, in a varied and specified patient population. Such a study is reported here. 


\section{Material and methods}

This report concerns a consecutive series of 33 patients with seizures of presumed or proven temporal lobe origin investigated with a combination of depth and subdural electrodes. In 26 patients an ictal onset zone in one temporal lobe was established by repeated ictal SEEG recording; in seven subjects a single site of electrographic seizure onset was not established. During the study period, the five years ending December 1991, the preoperative assessment protocol was, as far as possible kept constant, and operative procedures for temporal lobe resections changed little.

During the same period, 269 patients with medically intractable epilepsy were assessed for surgery. Forty one had hemispherectomy or partial callosal section, following a protocol different from that for possible local resections. All of the remaining 228 had wake and sleep EEGs. Detailed neuropsychological assessment was performed in all subjects, and a carotid amytal test (following the procedure described by Powell et $a l^{13}$ ) in all those considered for temporal resections. Ictal scalp recordings were performed in 199, in seven with sphenoidal, and in 192 with foramen ovale electrodes. Forty-eight had more invasive recordings with at least subdural electrodes, and all in the present study group were investigated by SEEG, with a combination of subdural and depth electrodes (table 1). All 33 study group subjects had undergone a Wada test and had temporal lobe orientated CT scans, nine had been investigated by MRI.

Of the 180 candidates for resection in whom more invasive recording was not considered to be indicated, 140 had resective surgery, and 30 were deemed inoperable. Ten withdrew themselves from the preoperative assessment programme.

The algorithms for selecting patients for subdural or depth (SEEG) recording are summarised in tables $2 \mathrm{a}-\mathrm{c}$.

\section{Assessment of scalp EEG and foramen ovale telemetry}

The EEG and telemetry studies used the Maudsley electrode placement system, which resembles the 10-20 system but provides more extensive cover, particularly of the anterior temporal region. ${ }^{14}$ Sleep recording was performed in all subjects, induced by sodium

\begin{tabular}{lr} 
Table $1 \quad$ Patient population & \\
\hline Assessed & 269 \\
Callosotomy/hemispherectomy & 41 \\
Assessed for local resection & 228 \\
Telemetry performed $(\mathrm{n}=199)$ & 192 \\
$\quad$ with foramen ovale electrodes & 7 \\
$\quad$ with sphenoidal electrodes & 140 \\
Subdural/depth recording not performed ( $\mathrm{n}=180)$ & 30 \\
$\quad$ operated & 10 \\
inoperable & 15 \\
patient withdrew from programme & 15 \\
$\quad$ extratemporal ictal onset zone & 7 \\
$\quad$ cps of presumed temporal origin $(\mathrm{n}=33:$ study group) & \\
$\quad$ no single ictal onset zone found & \\
$\quad$ single temporal ictal onset zone & 26 \\
\hline
\end{tabular}

quinalbarbitone, unless they slept spontaneously. A localising focus of epileptiform activity was considered to be present if spikes or spike wave activity occurred with a consistent maximum at a single electrode or adjacent electrodes, exceeding the discharge rate at any other site in a ratio of at least $4 / 1$.

Assessment of foramen ovale (FO) telemetry, for purposes of this study was confined to ictal events. A localised electrographic seizure onset was considered to have been detected if the initial changes (spikes, spike-and-wave, or a new rhythmic activity of theta, alpha or beta frequency) were consistently of maximum amplitude over one lobe of the brain.

Table $2 a$ General criteria for admission to preoperative assessment programme

1 Reliable diagnosis of intractable epilepsy:Attacks are epileptic, no pseudoseizures

Failure of appropriate medication

Patient is compliant

2 Seizures of such a frequency and nature that they are disabling, having regard to the patient's life style

3 No other contraindication, for example, a coagulation defect

Table $2 b$ Criteria for investigation for resective surgery and/or multiple subpial transection

1 Partial seizures, whether or not secondarily generalised

2 Does not meet criteria for hemispherectomy:-

a) Long-standing unilateral hemispheric damage

evidenced by hemiplegia and brain-imaging changes

b) Partial seizures, whether or not secondarily generalised apparently arising from the diseased hemisphere

3 Full scale IQ not less than 70

4 Age not more than 55 years

5 Patient has the emotional resources to withstand the procedures, including the possibility of being found inoperable

\section{Table 2c Specific criteria for subdural or SEEG recording}

In addition to meeting general admission requirements and specific criteria for assessment with a view to resective surgery, patients selected for invasive recording satisfy all of the following:-

A) The patient does NOT meet any of the following

A) The patient does NOT meet any of the following subdural or SEEG recording:-

1 (For lesionectomy) A discrete cerebral lesion, demonstrated by imaging,

concordant with

Seizure pattern

Site of interictal EEG discharges

Abnormalities of background interictal EEG and not

Neuropsychological findings.

At an eloquent site which demands functional mapping.

2 (For temporal resection) A consistent, single, temporal site of electrographic seizure onset, at or preceding clinical onset on foramen ovale telemetry,

concordant with

Seizure pattern

Abnormalities of background EEG

Brain imaging findings

Neuropsychological findings.

B) The patient does NOT show the following features which would exclude resective surgery:-

3 Predominantly generalised interictal EEG discharges in the absence of a discrete lesion on neuroimaging.

4 Independent sites of electrographic seizure onset demonstrated in more than one lobe by telemetry with foramen ovale electrodes.

5 Generalised epileptiform discharges at or preceding clinical seizure onset.

6 A probable site of seizure onset, which cannot be resected without unacceptable complications, and is considered unsuitable for multiple subpial transection. 
Introduction of electrodes and recording

The electrode technology employed is described in detail by Van Veelen et al. ${ }^{15}$ In brief, all electrodes were inserted through bilateral fronto-central trephine holes. Those placed in the subdural space had seven $5-\mathrm{mm}$ contacts on $10 \mathrm{~mm}$ centres and were of two types: thin $(0.3 \mathrm{~mm})$ flexible bundles, and flat "reeds" $3 \mathrm{~mm}$ in width (considerably narrower than conventional subdural strips) which were compliant in only one dimension and therefore sometimes easier to insert in the required direction. From 7 to 10 subdural bundles or reeds were placed under fluoroscopic control, in all subjects, including a minimum of one mid-temporal, one lateral frontal and one fronto-polar-orbital placement on each side.

The procedure for placement of depth electrodes differed from the method of Van Veelen et $a l,{ }^{15}$ in that CT controlled stereotaxy was used. Targets within mesial temporal structures were identified using CT scans orientated along the axes of the temporal horns, the gantry being tilted, typically at $20^{\circ}$, from the horizontal. Temporal target coordinates were calculated taking account of the angle of the gantry during imaging. ${ }^{16}$ Six-contact depth electrode bundles, 4 to 8 in number, were implanted bilaterally, through the same fronto-central trephine holes as were used for the subdural electrodes. They were placed in amygdala, anterior and/or posterior hippocampus and, as indicated, tangentially through the medial frontal cortex and anterior cingulum to the gyrus rectus.

Recording started when the patient had recovered from the operation three to seven days following implantation and continued for up to three weeks, mean 8.6 days. Antiepileptic medication was reduced or withdrawn in all cases. Cable telemetry of 32 or 64 channels was used (Telefactor MODAC or Beekeeper systems) with simultaneous video monitoring. During the last 18 investigations of the series automatic seizure recognition was employed using an algorithm based on that of Gotman ${ }^{17}$ (Telefactor SeZac system).

\section{Surgery and assessment of outcome}

Those patients in the study group who were treated surgically had either en bloc temporal lobe resection or amygdalohippocampectomy by the trans-Sylvian route. ${ }^{18}$ Outcome was assessed by the system of Engel, ${ }^{1}$ which divides seizure relief into four grades (each with two to four subgroups, a-d), and grade 5 , fatality. In brief: grade 1 seizure-free; 2 rare seizures; 3 worthwhile improvement ( $75 \%$ seizure reduction or prolonged seizurefree intervals of more than 2 years); 4 unimproved; 5 died. For purposes of the present analysis, grades 1 and 2 are regarded as a successful outcome.

\section{Results}

The 48 patients investigated with subdural or depth recording included 15 with a proven extra-temporal onset, which had been expected before SEEG. They are excluded from the study population as they do not form an homogeneous group, and are too few to analyse as sub-groups. The study group is therefore restricted to the patients with a presumed temporal seizure onset, and reasons for undertaking invasive recording will be considered in this sample of 33. Results concerning the utility of SEEG and comparison of SEEG with other data relate only to the 26 subjects with a single proven temporal ictal onset zone.

\section{Reasons for intracranial recording}

The case records were reviewed to determine what considerations had led to SEEG in these 33 patients (table 3 ). None met criteria 1-6 of table 2c. Seven with localised radiological abnormalities (two frontal, one thalamic, one parietal, one occipital and two temporal) failed to meet criterion 1 (for lesionectomy), as the site of the demonstrated lesion was not concordant with other evidence. The remainder failed criterion 2 (consistent temporal onset) because of:- a) Bilateral electrographic seizure onset on FO telemetry. ${ }^{14} \mathrm{~b}$ ) "Delayed electrographic onset", ${ }^{12}$ that is, the appearance during foramen ovale telemetry of clinical ictal phenomena before the first localised electrographic change (including an electrodecremental event or other "non-epileptiform" change). c) "Cross-over" that is, rapid appearance of bilateral or contralateral discharges, within 1 second of an apparently lateralised seizure onset at scalp or foramen ovale electrodes. d) Apparent extra-temporal electrographic onset. ${ }^{5}$ e) Non-concordance between electrographic ictal onset and consensus of other evidence. ${ }^{10} f$ ) Clinical seizure pattern non-concordant with consensus of other evidence. ${ }^{3}$ Multiple reasons were cited in 10 patients.

\section{Localisation of seizure onset by SEEG}

Detailed SEEG findings will be presented only in the 26 patients with reliably localised temporal ictal onset. Two hundred and sixty six seizures were captured in these subjects; sites of ictal onset on SEEG are summarised in table 4.

Fourteen patients showed, in addition to ictal onsets at one main site, a minority of seizures starting at adjacent electrodes in the same temporal lobe ("minor sites" in table 4).

Table 3 Reasons for selection for SEEG; see criteria 1-2 of table 2c. No patient was clearly unsuitable for resective surgery by criteria 3-6. These reasons are not mutually exclusive; 10 patients failed on multiple criteria

Reasons for selection for SEEG
1 Lesion on imaging not concordant with consensus of
clinical, psychological and electrophysiological
evidence
2 Ictal onset on telemetry:-
not located:
$\quad$ bilateral onset
rapid "cross-over"
"delayed onset"
not concordant with other evidence
not temporal
3 Clinical findings not concordant with other evidence


Table 4 Sites of seizure onset in SEEG.

\begin{tabular}{|c|c|c|c|}
\hline & \multicolumn{3}{|c|}{ Sites of onset } \\
\hline & Main & Minor & Subclinical \\
\hline Amygdala & 2 & 1 & 0 \\
\hline Anterior hippocampus & 11 & 6 & 9 \\
\hline Posterior hippocampus & 2 & 1 & 1 \\
\hline Anterior and posterior hippocampus & 1 & 0 & \multirow{2}{*}{$\begin{array}{l}0 \\
4\end{array}$} \\
\hline Hippocampus and amygdala & 5 & 1 & \\
\hline Lateral temporal & 2 & 1 & 1 \\
\hline Lateral temporal and hippocampus & 1 & 0 & \multirow[b]{2}{*}{1} \\
\hline Parietal and posterior hippocampus & 1 & 1 & \\
\hline Amygdala, anterior hippocampus & & & \multirow{4}{*}{$\begin{array}{l}0 \\
0\end{array}$} \\
\hline and lateral temporal & 1 & 0 & \\
\hline Frontal and anterior hippocampus & 0 & 1 & \\
\hline Frontal & 0 & 2 & \\
\hline
\end{tabular}

Seizure onset appeared to be localised to a single contact in 18 patients. The initial ictal discharge was confined to the anterior hippocampus in 11 and detected simultaneously in anterior hippocampus and amygdala in five. Anterior hippocampal onsets of some or all seizures occurred in 19 subjects, amygdaloid in eight. There was an initial lateral neocortical involvement in the majority of seizures in five patients, three of whom showed a regional onset in neocortex and hippocampus.

The initial discharge consisted of fast activity at 16 to approximately $50 \mathrm{~Hz}$ in 15 patients, and of a rhythmic build up of spikes starting at less than $13 \mathrm{~Hz}$ in six. Slow periodic spikes preceding a faster rhythmic ictal discharge, as described by Spencer et al, ${ }^{19}$ were rare, and occurred in a majority of seizures in only two patients, both with a regional seizure onset including neocortex. Neuropathological findings are now available in 19 patients: in 13 out of 15 with initial fast activity a clear abnormality was found, whereas specific pathology was present in only one of four patients who did not show this type of ictal onset, a finding in accordance with the observations of Spencer et al. ${ }^{19}$

Three subjects suffered from one to three seizures arising contralateral to the main ictal onset zone (not included in the analysis above). In all instances, these occurred late in the course of the investigation, several days after antiepileptic drug withdrawal, and within 30 minutes of a preceding seizure, the postictal electrophysiological sequelae of which had not yet resolved.

Sixteen patients exhibited sub-clinical electrographic seizures, which corresponded in both topography and electrographic pattern

Table 5 Lateralisation of interictal and ictal discharges with respect to seizure onset in $S E E G$

\begin{tabular}{|c|c|c|c|c|}
\hline & \multicolumn{4}{|c|}{ Lateralisation with respect to ictal SEEG } \\
\hline & \multirow{2}{*}{$\begin{array}{l}\text { Predominantly } \\
\text { ipsilateral }\end{array}$} & \multirow{2}{*}{$\begin{array}{l}\text { Not } \\
\text { lateralised } \\
\text { or absent }\end{array}$} & \multicolumn{2}{|l|}{ Contralateral } \\
\hline & & & Predominant & Exclusive \\
\hline \multicolumn{5}{|l|}{ Interictal } \\
\hline Subdural discharges & 7 & 11 & 8 & 0 \\
\hline Depth discharges & 19 & 1 & 6 & 0 \\
\hline & 16 & 4 & 4 & 2 \\
\hline \multirow{4}{*}{$\begin{array}{l}\text { Mid-ictal (depth and } \\
\text { subdural electrodes) } \\
\text { Late ictal (depth and } \\
\text { subdural electrodes) } \\
\text { Postictal (depth and } \\
\text { subdural electrodes) }\end{array}$} & 14 & 4 & 4 & 4 \\
\hline & 10 & 8 & 4 & 4 \\
\hline & & & & \\
\hline & 3 & 18 & 1 & 4 \\
\hline
\end{tabular}

to the initial events of overt seizures at the ictal onset zone. Thirteen of these subjects each exhibited over 50 such episodes in 2-21 days recording.

Subdural electrodes were involved at seizure onset in five patients. Discharges of mesial temporal origin propagated first to the ipsilateral neocortex in 11 subjects but to the contralateral convexity (via the hippocampus) in six. This early propagation to contralateral subdural contacts occurred consistently in two patients, and in the majority of seizures of four subjects (table 5). In three subjects subdural discharges appeared simultaneously over both hemispheres, following an early propagation to the contralateral hippocampus. In one patient the ictal discharges were confined to depth electrodes. Thus lateralisation relying on the first ictal events detected at subdural electrodes would have been incorrect in six patients and impossible in four.

Mid-ictal discharges at the stage of maximum generalisation, if any, and late ictal discharges in the last 10 seconds of the seizure, were with the exception of one patient, generally present at both subdural and deep electrodes, predominating ipsilateral to seizure onset in 14 out of 26 subjects (table 5). In four patients, following an early propagation to the contralateral mesial temporal structures, the subsequent mid- and late ictal discharges showed a consistent contralateral preponderance. Postictal slowing was correctly lateralising to the side of seizure onset in only three subjects, and usually contralateral in five.

Interictal epileptiform activity appeared bilaterally at deep electrodes in all patients: this showed a clear lateralised preponderance in all but one subject, and was maximal ipsilateral to the site of seizure onset in 19, contralateral in six. Lateralising interictal subdural discharges, by contrast, were present in only 15 patients, with a maximum ipsilateral to seizure onset in only seven.

Evidence of site of seizure onset before SEEG

In this group of patients, lateralisation of the site of SEEG seizure onset by less invasive means was conspicuously unsuccessful (table 6 ). The carotid amytal test showed the greatest reliability, the onset zone being in the more dysfunctional hemisphere in 10 instances and contralateral in only one. Lateralising abnormalities on general neuropsychological assessment were uncommon, but were also fairly reliable (six implicating the ipsilateral, and one the contralateral hemisphere).

Lateralisation by routine wake and sleep EEG was random: 18 patients had lateralised discharges, but the lateralisation agreed with the findings on SEEG in only nine. Lateralisation by foramen ovale telemetry agreed with SEEG findings in 10 patients and disagreed in five. These five failures of lateralisation by $\mathrm{FO}$ telemetry were explicable by the SEEG findings. This group included both of the patients with an ictal onset zone confined to the amygdala, which has a closed 
Table 6 Lateralisation by non-invasive methods with respect to seizure onset in SEEG

\begin{tabular}{lccl}
\hline & \multicolumn{2}{l}{ Lateralisation by non-invasive methods } \\
\cline { 2 - 4 } & Ipsilateral & Not lateralised & Contralateral \\
\hline Clinical ictal pattern & 6 & 16 & 4 \\
CT and/or MRI & 3 & 23 & 0 \\
Psychometrics & 6 & 19 & 1 \\
Carotid amytal test & 10 & 15 & 1 \\
Non-invasive neurophysiology: & 9 & 8 & 9 \\
Wake and sleep EEG & 10 & 11 & 5 \\
FO telemetry & & NA & 0 \\
Concordant findings: & 4 & NA & 1 \\
Telemetary and EEG & 4 & NA & 2 \\
Clinical and EEG & 3 & NA & 0 \\
Clinical and telemetry & 3 & NA & 1 \\
EEG and amytal & 4 & NA & 0 \\
Telemetry and amytal & 3 & &
\end{tabular}

electrical field not detectable from without. ${ }^{20}$ All five showed rapid propagation to the contralateral hippocampus. FO telemetry had shown clinical ictal onset before the first apparent electrographic changes and rapid cross-over (see above). These, indeed, were the features which had led to selection for SEEG.

Concordant lateralisation on interictal EEG and FO telemetry was found in only four instances, but agreed with SEEG in all of these. Clinical and EEG lateralisation were concordant in five subjects, and disagreed with SEEG findings in one of these.

Lateralising abnormalities on neuroimaging were found ipsilateral to seizure onset in three instances, but implicated an extra-temporal site in all cases.

\section{Outcome of surgery in relation to electrophysiological findings}

Twenty one patients have undergone surgery, and one to four years follow up have been completed in 15. Two were deemed inoperable despite localisation of ictal onset on neuropsychological grounds: the contralateral hemisphere did not support memory on the Wada test, precluding en bloc temporal lobe resection, and the onset zone involved lateral neocortex so that amygdalohippocampectomy was considered inappropriate. One patient has refused operation and a decision has been deferred in another, due to spontaneous remission. One has asked to defer a planned temporal resection. In patients with at least one year's follow up, the median outcome (following the classification of $\mathrm{Engel}^{17}$ ) is $2 b$.

The sequence of electrophysiological findings and eventual outcome are summarised in table 7. Interictal epileptiform activity in the wake and sleep EEG was unifocal or showed a clearly lateralised preponderance in 25 patients, but this agreed with SEEG lateralisation, where achieved, in only $9 / 18$. Ictal findings on FO telemetry were concordant with interictal scalp EEG in six instances; SEEG was confirmatory in four, but failed to identify a single ictal onset zone in two. FO telemetry led to a reversal of lateralisation in nine patients. SEEG confirmed this revised lateralisation in five, led to a further reversal in three, and was non-localising in one. In 10 patients with lateralising EEG findings telemetry showed bilateral changes at seizure onset. An ictal onset zone was identified by SEEG in six of these. In the eight patients without lateralising interictal EEG discharges, telemetry showed a unilateral onset in three, confirmed by SEEG in only one, and reversed in two. In five patients neither interictal EEG nor telemetry provided evidence of lateralisation, SEEG identified the ictal onset zone in all of these, but in the three who have so far completed one year's follow up after operation the results were poor.

\section{Discussion}

The utility of SEEG

The patients in our study were selected for SEEG specifically because the site of seizure onset was considered not to have been reliably determined by other means. Thus the

Table 7 Sequence of electrophysiological localisation and surgical outcome

\begin{tabular}{|c|c|c|c|c|}
\hline $\begin{array}{l}\text { Interictal } \\
\text { scalp EEG }\end{array}$ & $\begin{array}{l}\text { Foramen ovale } \\
\text { telemetry }\end{array}$ & SEEG & $\begin{array}{l}\text { Surgical } \\
\text { outcome }\end{array}$ & Pathology \\
\hline \multirow{5}{*}{$\begin{array}{l}25 \\
\text { lateralised }\end{array}$} & \multirow{2}{*}{$\begin{array}{l}6 \\
\text { laterality } \\
\text { confirmed }\end{array}$} & 4 confirmed & 1a $4 \mathrm{a} \mathrm{EO}$ & MTS CD MTS ? \\
\hline & & 2 non- & N N & ? ? \\
\hline & \multirow[t]{2}{*}{$\begin{array}{l}9 \\
\text { laterality } \\
\text { reversed }\end{array}$} & $\begin{array}{l}5 \text { confirmed } \\
3 \text { reversed } \\
\text { again }\end{array}$ & $\begin{array}{l}1 \mathrm{a} 3 \mathrm{a} 1 \mathrm{~b} \\
\mathrm{EN} \\
2 \mathrm{a} 2 \mathrm{~b} E\end{array}$ & $\begin{array}{l}\text { MTS MTS NS } \\
\text { MTS ? } \\
\text { MTS MTS NS }\end{array}$ \\
\hline & & $\begin{array}{l}1 \text { non- } \\
\text { localised }\end{array}$ & $\mathbf{N}$ & ? \\
\hline & $\begin{array}{l}10 \\
\text { diffuse } \\
\text { bilateral }\end{array}$ & $\begin{array}{l}6 \text { now } \\
\text { localised } \\
4 \text { non } \\
\text { localised }\end{array}$ & $\begin{array}{l}2 c 51 \mathrm{~A} \\
\text { NNE } \\
\text { NNN N }\end{array}$ & $\begin{array}{l}\text { NS MTS MTS } \\
\text { ?? CT } \\
\text { ?? ?? }\end{array}$ \\
\hline \multirow{2}{*}{$\begin{array}{l}8 \text { not } \\
\text { lateralised }\end{array}$} & $\begin{array}{l}3 \\
\text { lateralised }\end{array}$ & 1 confirmed & $4 \mathrm{~b}$ & MTS \\
\hline & $\begin{array}{l}5 \text { not } \\
\text { lateralised }\end{array}$ & $\begin{array}{l}2 \text { reversed } \\
5 \text { now } \\
\text { lateralised }\end{array}$ & $\begin{array}{l}1 \mathrm{bE} \\
3 \mathrm{a} 3 \mathrm{a} 3 \mathrm{a} \\
\text { NE }\end{array}$ & $\begin{array}{l}\text { MTS MTS } \\
\text { NS MTS NS } \\
\text { ? MTS }\end{array}$ \\
\hline
\end{tabular}

*Lateralisation in agreement with scalp EEG in 2.

Surgical outcome:

$\mathrm{N}$ : no operation to be performed (8 not localised, 1 refused, 1 remitted, 2 inoperable on neuropsychological grounds); O: awaiting operation; E: early postoperative, less than 1 year's follow up; 1-5 outcome grades following Engel (1987).

Pathology:

MTS: mesial temporal sclerosis; CD: cortical dysplasia; CT: low grade cerebral tumour; ? not available (not operated); NS: no significant specific pathology found. 
lack of agreement between localisation by SEEG and by less invasive methods is not a reflection of the general unreliability of the latter, but evidence rather of the relevance of the selection criteria employed. No single non-invasive method of assessment achieved correct lateralisation in more than one third of operable patients; various combinations of assessments appeared no more reliable.

The findings of SEEG are traditionally taken to be the "gold standard" of preoperative localisation. ${ }^{21-23}$ However, locating ictal onset does not guarantee a successful outcome of technically adequate surgery. Determining the ictal onset zone and studying early discharge propagation does not provide an objective means of identifying the hypothetical "epileptogenic zone", the removal of which is both necessary and sufficient to abolish seizures. ${ }^{24}$ In some cases there may exist no zone meeting this definition, the patient being inherently inoperable, for such reasons as diffuse cortical hyperexcitability. Development of criteria for identifying such subjects should be an urgent priority. Conversely, surgical success is not proof of accurate electrophysiological localisation. It is entirely possible that disconnection of seizure propagation pathways during surgery based on an incorrect identification of the onset zone may sometimes give a good result.

In terms of its immediate, limited objectives, SEEG may be considered successful if a single, circumscribed ictal onset zone is found, and if surgical resection is followed by worthwhile seizure relief and/or results in removal of tissue containing a discrete lesion. Seven SEEGs were clearly unsuccessful by these standards, as an ictal onset zone was not located, but may be considered to have been of value in establishing inoperability and hence avoiding inappropriate surgery. In the 18 patients so far operated 16 meet the above criteria of successful SEEG; two, however, have achieved only a grade 3 a outcome after removal of essentially normal tissue. These two cases arguably represent failures of localisation by SEEG, but have no apparent distinguishing clinical or electrographic features.

The contribution of SEEG to preoperative assessment in this study group may be summarised as follows:-

1 Confirmatory of previous localisation-10 $(30 \%)$

2 Erroneous localisation corrected-5 (15\%)

3 Localisation achieved-11 (33\%)

4 Inappropriate operation avoided-7 (21\%)

Spencer $^{25}$ reviewed published reports of 178 patients who had depth recording and classified the comparative EEG and SEEG findings into five categories. Our four groups approximate to her categories $1,2,3$, and 4 combined with 5 , which respectively comprised $30 \%, 9 \%, 36 \%$ and $25 \%$ of the total. Spencer suggested that such results indicated that the number of operative candidates can be increased by $36 \%$ (category 3 ) through the use of SEEG. However, in their own material Spencer $e t a^{26}$ failed to localise ictal onset in 16 out of 31 patients (52\%) (Spencer's groups 4 and 5). Neither study analyses the proportions of patients undergoing invasive recording and the implications of selection criteria. As we performed SEEG selectively in a small minority of the 269 patients forming the parent population and 181 had surgery without SEEG, the percentage increase in operability amounted to only $6 \%$. Alternatively, the proportion of the 269 referrals in whom the procedure could be considered to have improved preoperative assessment (our groups 2, 3, and 4) makes up $8.6 \%$.

\section{PARTICULAR CONSIDERATIONS IN \\ LOCALISATION}

Subclinical seizures

Sperling and O'Connor ${ }^{27}$ reported subclinical electrographic seizures in 16 patients with complex partial seizures of temporal origin. The sites of onset of subclinical and overt seizures were coincident in 14 , but as this was not the case in two, they concluded that subclinical seizures did not reliably indicate the origin of the overt attacks. In our material there were also 16 patients with subclinical seizures, but here there was complete agreement with the localisation of clinical episodes. Only three patients without subclinical seizures have so far had surgery, with outcome grades of $3 A, 3 A$, and $4 B$, whereas the median outcome in the subclinical seizure group is $2 b$. There is thus a trend in accordance with a finding of Sperling and $\mathrm{O}^{\prime}$ Connor, ${ }^{27}$ that patients without subclinical seizures have less favourable results.

\section{Anomalous site of onset}

Three patients showed a reversal of laterality of mesial temporal seizure onset after antiepileptic drug (AED) withdrawal and when a second seizure supervened within the period of postictal electrographic change up to 30 minutes following a previous attack. Subsequent seizures after a greater interictal interval, both before and after restoration of medication, showed the habitual pattern. The typical seizures of all three patients rapidly propagated to the contralateral hippocampus. It remains uncertain how far the inter-seizure interval or the withdrawal of medication was the determining factor in the occurrence of atypical seizures. Medication withdrawal is generally accepted as a satisfactory means of promoting seizure occurrence during telemetry: apart from an increased tendency to secondary generalisation the seizures follow their habitual pattern. ${ }^{28}{ }^{29}$ Engel and Crandall ${ }^{30}$ report a single patient with an anomalous seizure onset following AED withdrawal; arguably three further instances can be claimed here. However, in our patients (unlike that of Engel and Crandall) a consistent relationship of atypical onset to a short preceding interictal interval provided grounds for regarding these attacks as anomalous and it was decided to discount them for purposes of preoperative assessment. This decision appeared justified by surgical outcome (grade 1 in two patients, grade 2 in one). 
Interictal SEEG discharges

Interictal discharges were present bilaterally in all SEEG recordings, but when clearly predominant on one side did not reliably lateralise the ictal onset zone, this was particularly so at subdural contacts; lateralisation of the side of onset at subdural electrodes was also of no predictive value. These results agree with the experience of some other investigators ${ }^{31-33}$ but conflict with the findings of Lüders $e t a l^{44}$ who noted a high rate of concordance between ictal and interictal findings in an, admittedly more extensive, array of subdural electrodes. Whether or not this is of practical consequence will depend on the criteria used to plan the resection. In centres performing tailored procedures intended to include, so far as possible, the irritative zone, the delineation of this is obviously important.

\section{Subdural placements}

In ictal recordings too, subdural placements failed to provide reliable lateralisation. There was initial subdural involvement in a focal or regional onset in five subjects and early appearance of discharges at ipsilateral subdural electrodes in 11 ; in the remaining six, propagation occurred first to the contralateral surface contacts in most or all seizures. The failure rate of localisation by subdural electrodes is similar to that in comparative studies of simultaneous depth and subdural recording, ${ }^{2735}$ but again in disagreement with the conclusions of Lüders $e t$ al ${ }^{34}$ using subdural electrodes only. This result also conflicts with the findings of Spencer $e t a l^{36}$ in eight patients with depth and bilateral subdural electrodes; they did not observe propagation to contralateral neocortex before ipsilateral involvement. The high incidence of false lateralisation by subdural electrodes is similar to the error rate with multipolar FO contacts, which may be considered to provide a more extensive mesial temporal subdural coverage than can be achieved either by the subdural reeds which we employed or by conventional strip electrodes inserted by a lateral approach. The purpose of FO recording was to reduce the need for SEEG, and this is likely to have favoured the selection of patients without early mesial temporal subdural discharges.

\section{SELECTION FOR SEEG}

Concerning the criteria of selection for SEEG, three queries arise:

1 Was SEEG performed unnecessarily in patients in whom reliable localisation had already been achieved?

2 Was SEEG performed to no useful purpose in patients in whom it could have been predicted that: a) no single ictal onset zone would be found; b) surgical outcome would be poor, or c) operation would be contraindicated on neuropsychological grounds?

3 Was SEEG withheld in patients who would have benefited from its use?

Addressing these issues

1 Localisation without SEEG

Concordance between any two non-invasive assessments was found rarely (table 6) and did not appear to offer reliable evidence of lateralisation. Again, this is unremarkable as lack of consistency between different assessments was a major factor in selection for SEEG. The highest specificity may be claimed for the finding, uncommon in this group, of agreement between routine EEG and telemetric lateralisation. The reasons for proceeding to SEEG in the four subjects with concordant interictal and ictal EEG findings were, however, not exceptional: delayed electrographic ictal onset in three, and frontal localisation of EEG discharges with clinical disparity in one.

\section{2a Failure of SEEG localisation}

In seven patients (21\%) no single ictal onset zone was found, and the procedure conferred no benefit beyond avoidance of a possibly inappropriate operation. This is a proportion similar to the $18 \%$ reported by Spencer. ${ }^{25}$ The most conspicuous features of this group were that five of the seven had atrophic lesions on neuroimaging (mesial temporal in two, thalamic in one, frontal in two), only one had a unilateral EEG focus, and none had lateralising findings on telemetry. In all but the patient with a thalamic abnormality, the intracranial electrode array included contacts in the region indicated by imaging, but seizure onset was not located at this site nor elsewhere. By contrast, of 26 patients with a demonstrable ictal onset zone, only one had a concordant localised abnormality on imaging (unilateral dilatation of one ventricular horn, compatible with MTS), 18 had lateralised EEG foci and 15 lateralised ictal onset on telemetry (albeit incorrectly lateralised in nine and five instances, respectively). On such small numbers it would be premature to draw any conclusion. However, these findings may suggest that lack of lateralisation by both interictal EEG and ictal FO telemetry, in the face of clear radiological abnormalities may be an adverse feature in selection for SEEG.

\section{$2 b$ Poor surgical outcome}

The number of operated patients with at least one year's follow up is too small to permit identification of any group in whom an unfavourable result could have been predicted and SEEG avoided. However, in the three subjects without localisation by scalp EEG or foramen ovale telemetry, results were poor and in two of the three no significant pathology was found. On these small numbers there is no convincing evidence of an identifiable group in whom SEEG was superfluous, but in a larger prospective series it would be worth testing the present trends which suggest that when EEG and telemetry provide concordant localisation, SEEG is confirmatory. When both are negative, SEEG too may fail to locate the ictal onset zone, even in the presence of abnormalities on imaging (see 2a above), and if the site of seizure onset is apparently identified, operative results may yet be poor. 
2c Neuropsychological contraindication to surgery In two patients no operation was performed because the Wada test suggested a high risk of postoperative memory deficit. However, this could not have been predicted before SEEG which lateralised the site of ictal onset to a hemisphere required to support memory, and demonstrated a neocortical onset, precluding the use of selective amygdalohippocampectomy to preserve memory.

Even with hindsight, there are no apparent criteria by which patients who did not require, or could not benefit from, SEEG could have been identified.

Obviously, this study cannot address the third issue: the question of whether a greater proportion of patients could have benefited from SEEG. This will be difficult to resolve except possibly by comparing these findings with a similar analysis of clinical material from centres using less conservative selection criteria and therefore employing SEEG in a larger proportion of patients.

1 Engel J. Outcome with respect to epileptic seizures. In: Engel J, ed. Surgical treatment of the epilepsies. New York: Raven Press, 1987:553-71.

2 Rasmussen T. Cortical resection in the treatment of focal epilepsy. In: Purpura DP, Penry JK, Walter RD, eds. Neurosurgical management of the epilepsies. Adv Neurol, 1975:8:139-54.

3 Davidson S, Falconer MA. Outcome of surgery in 40 children with temporal lobe epilepsy. Lancet 1975;1: 1260-3.

4 Polkey CE. Prognostic factors in selecting patients with drug-resistant epilepsy for temporal lobectomy. In: Rose FC, ed. Research progress in epilepsy. London: Pitman, 1983:500-6.

5 Wright GDS, Oxbury JM, Fenwick P, Oxbury SM, Adams CBT. The possible need for intracranial EEG in
surgery for temporal lobe epilepsy. Acta Neurol Scand 1990;82:20.

6 Bloom D, Jasper H, Rasmussen T. Surgical therapy in patients with temporal lobe seizures and bilateral EEG abnormality. Epilepsia 1959;1:351-65.

7 Falconer MA. Anterior temporal lobectomy for epilepsy. In: Logue V, ed. Operative surgery, vol 14. Neurosurgery. In: Logue V, ed. Operative surgery,

8 Delgado-Escueta AV, Walsh GO. The selection process for surgery of intractable complex partial seizures: surface EEG and depth electrography. In: Ward AA, Penry 1983:295-300.

9 Morris HH, Kanner A, Lüders H, Murphy D, Dinner DS, Wyllie E, Kotogal P. Can sharp waves localized at the sphenoidal electrode accurately identify a mesiotemporal epileptogenic focus? Epilepsia 1989;30:532-9.

10 Engel J, Crandall PH, Brown WI. Consistent false lateralisation of seizure onset with sphenoidal and scalp telemetered ictal EEG recordinos in two parients with partial complex epilepsy. Electroencephalogr Clin Neurophysiol 1980;50:60.

11 Spencer SS, Williamson PD, Bridgers SL, Mattson RH, Ciccetti DV, Spencer DD. Reliability and accuracy of localization by scalp ictal EEG. Neurology 1985;35: 1567-75.

12 Engel J, Sherman DA. Overview: Who should be considered for surgical treatment? In: Engel J, ed. Surgical treatment of the epilepsies, 1993:23-34.

13 Powell GE, Polkey CE, Canavan AGM. Lateralisation of memory function in epileptic patients by use of the sodium amytal (WADA) technique. $\mathcal{f}$ Neurol Neurosurg Psychiatry 1987;50:665-72.

14 Margerison JH, Binnie CD, McCaul IR. Electroencephalographic signs employed in the location of ruptured intracranial arterial aneurysms. Electroencephalog Clin Neurophysiol 1970;28:296-306.

15 Van Veelen CWM, Debets RM, Van Huffelen AC, et al. Combined use of subdural and intracerebral electrodes in preoperative evaluation of epilepsy. Neurosurgery 1990;26:93-101.

16 Agbi C, Polkey CE. Calculation of coordinates for depth electrodes placed in temporal lobe structures visualized by oblique CT scan cuts. Br $f$ Neurosurg 1990;4: by oblique.

17 Gotman J. Automatic recognition of epileptic seizures in the EEG. Electroencephalogr Clin Neurophysiol 1982;54: $530-40$

18 Yasargil MG, Teddy PG, Roth P. Selective amygdalohippocampectomy. Operative anatomy and surgical technique. In: Symon L, ed. Advances and technical standards in neurosurgery vol, 12. Vienna: SpringerVerlag, 1985:93-123.

19 Spencer SS, Guimaraes P, Katz A, Kim J, Spencer D. Morphological patterns of seizures recorded intracranially. Epilepsia 1992;33:537-45.

20 Gloor P. Volume conductor principles: their application to the surface and depth electroencephalogram. In: Wieser H-G, Elger CE. eds. Presurgical evaluation of epileptics. Berlin: Springer, 1987:59-70.

21 Gloor P. Contributions of electroencephalography and electrocorticography to the neurosurgical treatment of the epilepsies. In: Purpura DP, Penry JK, Walter RD the epilepsies. In: Purpura DP, Penry JK, Neurosurgical management of the epilepsies. Adv Neurol 1975;8:59-106.

22 Rossi GF. Problems of analysis and interpretation of electrocerebral signals in human epilepsy: a neurosurgeon's view. In: Brazier MAB, ed. Epilepsy: its phenomena in man. New York: Academic Press, 1973:261-87.

23 Walter RD. Principles of clinical investigation of surgica candidates. In: Purpura DP, Penry JK, Walter RD, eds. Neurosurgical management of the epilepsies. Adv Neurol, 1975;8:49-58.

24 Lüders H, Awad, I. Conceptual considerations. In: Lüders H. Epilepsy surgery. New York: Raven Press 1991:57-62.

25 Spencer SS. Depth electroencephalography in selection of refractory epilepsy for surgery. Ann Neurol 1981;9: 207-14.

26 Spencer SS, Spencer DD, Williamson PD, Mattson RH. The localizing value of depth electroencephalography in 32 patients with refractory epilepsy. Ann Neurol 1982;12:248-53.

27 Sperling MR, O'Connor MJ. Auras and subclinical seizures: characteristics and prognostic significance. Ann Neurol 1990;28:320-8.

28 Spencer SS, Spencer DD, Williamson PD. Ictal effects of anticonvulsant medication withdrawal in epileptic patients. Epilepsia 1981;22:297-307.

29 Marciani MG, Gotman J, Andermann F. Patterns of seizure activation after withdrawal of antiepileptic medication. Neurology 1985;35:1537-43.

30 Engel J, Crandall P H. Falsely localizing ictal onsets with depth EEG telemetry during anticonvulsant withdrawal. depth EEG telemetry during

31 Lieb JP, Joseph JP, Engel J, et al. Sleep state and seizure foci related to depth spike activity in patients with temporal lobe epilepsy. Electroencephalogr Clin Neurophysiol 1980;49:538-57.

32 Lieb JP, Engel J, Gevins A, Crandall P. Surface and deep correlates of surgical outcome in temporal lobe epilepsy. Epilepsia 1981;22:515-38.

33 Engel J, Rausch R, Lieb JP, Kuhl DE, Crandall $P$ Correlation of criteria used for localizing epileptic foci in patients considered for surgical therapy of epilepsy. Ann Neurol 1980;9:215-24.

34 Lüders H, Hahn J, Lesser RP, Dinner DS, et al. Basal temporal subdural electrodes in the evaluation of patients with intractable epilepsy. Epilepsia 1989;30: patients

35 Van Emde Boas W, Brekelmans GJF, Velis DN, Van Huffelen AC, Van Veelen CWM. Comparison of depth and subdural intracranial electrodes in recording seizures of temporal and extratemporal lobe origin. Neurophysiol Clin 1992;22:45s.

36 Spencer SS, Williamson PD, Spencer DD, Mattson RH. Combined depth and subdural electrode investigation in uncontrolled epilepsy. Neurology 1990;40:74-9. 